\title{
Nicht mehr atypische Femurfrakturen unter Alendronat
}

\author{
Eine Alendronat-Therapie senkt bei postmenopausalen Osteoporose-Patientinnen das \\ Hüftfrakturrisiko. Allerdings sind als Nebenwirkungen unter anderem atypische Femur- \\ frakturen bekannt. Eine dänische Kohortenstudie untersuchte nun den Langzeiteffekt einer \\ Alendronat-Therapie auf das Frakturrisiko bei Patienten mit Osteoporose.
}

In die landesweite, offene Register- und Fall-Kontroll-Studie waren 61990 Frauen und Männer eingeschlossen, die zwischen 1996 und 2007 Alendronat einnahmen. Zu Beginn der Therapie waren sie zwischen 50 und 94 Jahren alt. Die Studienauswertung (Fallgruppe) enthielt die Inzidenz von subtrochantären Femurfrakturen (ST), Femurschaftfrakturen (FS) und Hüftfrakturen . Die Patienten aus der Kontrollgruppe, die keine Knochenbrüche hatten, wurden anhand von Geschlecht, Geburtsjahr und Beginn der Alendronat-Therapie mit jenen aus der Fallgruppe verglichen.

1428 der Studienteilnehmer hatten eine ST/FS (Inzidenzrate 3,4/1000 Personenjahre, 95\%-Konfidenzintervall 3,2-3,6) und 6784 eine Hüftfraktur (16,2/1000, [15,8-16.6]). Das Risiko für eine ST/FS war unter Langzeittherapie nicht erhöht ( $\geq 10$ Dosisjahre; adjustierte Odds Ratio $0,70 ;[0,44-1,11] ; p=0,13)$. Ebenso hatten aktuell therapierte Patienten kein höheres Risiko als vergangene Anwender (0,91; [0,79-1,06]; $p=0,22)$. Ein hohes Medikationsverfügbarkeitsverhältnis (>80\%) stand mit einer Senkung des Hüftfrakturrisikos $(0,73$; $[0,68-0,78]$; $\mathrm{p}<0,001)$ in $\mathrm{Zu}-$ sammenhang, ebenso wie eine kumulative Langzeitbehandlung (5-10 Dosisjahre: 0,$74 ;[0,67-0,83] ; \mathrm{p}<0,001 ; \geq 10$ Dosisjahre: 0,$74 ;[0,56-0,97] ; p=0,03)$.

Die Behandlung mit Alendronat $\geq 10$ Jahre reduzierte bei guter Therapieadhärenz das Hüftfrakturrisiko um 30\%. Das Risiko für subtrochantäre Femurfrakturen sowie Femurschaftfrakturen stieg dadurch nicht. Selbst wenn alle ST/FS bei den Alendronat-Anwendern atypisch waren, stünde das Nutzen-Risiko-Verhältnis zugunsten des verminderten Hüftfrakturriskos, so die Autoren .

Dr. rer.nat. Marion Rukavina, Berlin DOI 10.1055/s-0042-115041

Abrahamsen B et al. Risk of hip ... BMJ 2016; 353: i3365

\section{Kommentar aus der Praxis}

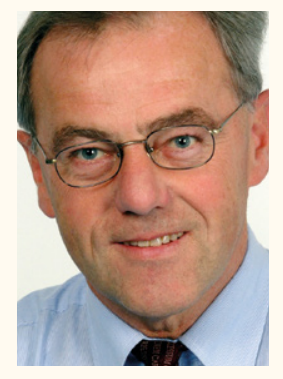

Prof. Dr. med. Reiner Bartl

Immer wieder wurden Bedenken geäußert, ob der verminderte Knochenumbau unter jahrelanger Bisphosphonat-Therapie zu einem überalterten, „eingefrorenen“, minderwertigen, spröden Knochen und damit vermehrt zu Mikrofrakturen („microdamages“) führt. Diese Einwände sind durchaus ernst zu nehmen und zu überprüfen. Alle bisherigen Langzeitstudien mit Bisphosphonaten (BP) haben diese Bedenken aber widerlegt. Die „American Society for Bone and Mineral Research“ (ASBMR) hat Kriterien für die Diagnose von atypischen BP-assoziierten Femurschaftfrakturen festgelegt. Dieser Frakturtyp wurde aber nicht nur unter Langzeittherapie ( $>5$ Jahre) mit oralen BP (5/10000 Patientenjahre), sondern auch bei Patienten, die kein BP einnahmen (1/10000 Patientenjahre), beobachtet. Wahrscheinlich sind die Patienten ohne Therapie in den Studien unterrepräsentiert. Ein kausaler Zusammenhang zwischen einer Langzeittherapie mit BP und diesem Frakturtyp konnte statistisch nie klar nachgewiesen werden.

Die vorliegende, neue dänische Kohortenstudie belegt - wie zu erwarten eine Reduktion des Risikos von Hüftfrakturen um 30\% und zeigt kein erhöhtes Risiko für atypische Femurschaftfrakturen. Trotz der extremen Sel- tenheit von atypischen Femurschaftfrakturen sollten vor allem unter Langzeittherapie mit BP deren typische Prodromi mit dem Patienten diskutiert werden. Typisch sind der Fraktur vorausgehende dumpfe, „tiefe“ Schmerzen im Bereich des Oberschenkels und des Gesäßes, häufig bilateral.

Ein einjähriges Aussetzen der Therapie („drug holiday“) nach 5 Jahren kontinuierlicher BP-Therapie ist generell empfehlenswert. Bei Patienten mit schwerer (T-score <-3) und manifester Osteoporose sowie bei Risikopatienten (Glukokortikoide, Aromatasehmmer) ist eine solche Pause allerdings nicht zu rechtfertigen.

Treten bilaterale, tiefe Schmerzen im Oberschenkel-, Hüft- und Gesäßbereich auf, wird folgende Diagnostik empfohlen: Röntgenbild, Skelett-Szintigramm oder MRT des betroffenen Femurs. Eine Osteomalazie mit Auftreten von Looserschen Umbauzonen muß mit der Bestimmung von Vitamin D, Kalzium und der alkalischen Phosphatase im Serum ausgeschlossen werden. Bei Nachweis von Frakturlinien oder Periostverdickungen, sollten die BP abgesetzt und Vitamin D (2000-3000 IE Vitamin $\mathrm{D}_{3}$ tgl.) gegeben werden. Weiterhin ist es ratsam, Stöße und Belastung des betroffenen Beines zu vermeiden. Liegt eine inkomplette Fraktur vor, wird in der Literatur eine prophylaktische intramedulläre Nagelung empfohlen.

Prof. Dr. med. Reiner Bartl Leiter des Osteoporosezentrums München am Dom

\section{Interessenkonflikt}

Der Autor gibt an, dass kein Interessenkonflikt besteht. 